\title{
A Phylogenetic and Genotyping Study of Bovine Herpesvirus Type 4 (BHV-4) in Turkey
}

\author{
B. Taylan KOÇ \\ Department of Virology, Faculty of Veterinary Medicine, Aydın Adnan Menderes University, \\ Işıklı, Efeler-Aydın, 09016, TURKEY
}

Received 2019-10-31 Accepted 2020-05-26

\begin{abstract}
Infertility and reproductive infections are the huge problems for the diary management throughout the world. Bovine herpesviruses act an enormous role in these complicated problems. Bovine Herpesvirus Type 1 (BHV-1) is the most outstanding herpesvirus causing genital and uterine tracts infections among other reproductive viral agents, however, Bovine Herpesvirus Type 4 (BHV-4) is also responsible in terms of similar symptoms and diseases. The main aims of the study are both to investigate the underlying potential presence of BHV-4 in subclinical uterine tract infection, and both to perform molecular and recombination analyses. A herd including 25 repeat breeder cows were investi-gated by BHV-4. Two out of them were determined BHV-4 infected after a series of Polymerase Chain Reactions (PCRs) tests which able to amplify partial Glycoprotein B (gB) and Thymidine Kinase (TK) gene regions. Obtained sequences were analyzed by using phylogenetic and recombinational software, and two Maximum Likelihood (ML) tree have been constructed. To results, novel Turkish BHV-4 sequences fell into Genotype I in both constructed Maximum Likelihood (ML) phylogenetic trees, however, no recombination evidence has been observed in relevant software. This report is one of the rare genotyping studies on BHV-4 from Turkey. This study showed that Turkish BHV-4 strains, as molecular, were highly probable to originate from European strains and might be observed in different clinical reflections. This suggests that BHV-4 should be deeply investigated by further molecular techniques and included in diagnostic panels for reproductive viruses.
\end{abstract}

Keywords: Reproductive Infection, Bovine herpesvirus Type 4 (BHV-4), Repeat-breeder, Infertility, Genotyping, Turkey

\section{Introduction}

Bovine herpesviruses (BHVs) are prominent viral agents of cattle all around the world, which mainly causes subclinical cases including respiratory and reproductive infections. Bovine Herpesvirus Type 4 (BHV-4) has been thought to predominantly cause genital diseases rather than other herpesviruses, although it was first isolated from the respiratory tract of a calf with relevant symptoms. ${ }^{1,2}$ BHV-4 taxonomically belongs to genus Rhadinovirus, subfamily Gammaherpesvirinae, family Herpesviridae. ${ }^{3}$
Pathogenetic mechanism of BHV-4 and its reflection on the clinical presentation is not widely known according to previous reports. ${ }^{1,4,5}$ In performed studies so far, it has been emphasized that the vast majority of BHV-4 cases has trigger subclinical infections which leading infertility problems and losses of milk yield. ${ }^{1,3,5}$ Genetic variability also might induce different pathogenesis, clinical diseases and latency determination, thus, molecular investigation of BHV-4 in infertile, repeat breeder animals have crucial importance in terms of dairy health. ${ }^{1,4-7}$

BHV-4 has double-stranded DNA in length of $144 \mathrm{~kb}$

\footnotetext{
* Corresponding author: B.T. Koç, E-mail: btkoc@adu.edu.tr, Tel: +90 256 2470700, Postal Adress: Department of Virology, Faculty of Veterinary Medicine, Aydın Adnan Menderes University, Işıklı, Efeler-Aydın, 09016, TURKEY
} 
which has many critical gene regions for protein expres- Bovine Herpesvirus Type-1 (BHV-1), Bovine sion. Glycoprotein B (gB) and Thymidine Kinase (TK) are Leukemia Virus (BLV), and all of them had been most significant gene regions and characterized by various reported negative.

molecular studies. ${ }^{6-8} \mathrm{gB}$ is more conserved gene region rather than TK, accordingly, has been used for general di-agnosis protocol. ${ }^{6,9}$ TK is preferred for molecular and phylogenetic studies, because of its close relationship with the host cell and being prone to molecular genetic changes. ${ }^{4,6}$ Three main genotypes based on TK, therefore, have been proposed by many researchers who have recently performed molecular and phylogenetic analyses in their papers. ${ }^{4,6,7,10}$ Genotype I has included Movar (33/63), reference strain from Europe, in which other sequences have been also Movarlike strains. Likewise, DN-599, reference strain of America, has fell into Genotype II which has other DN-599 like BHV-4 sequences. Genotype III has been consisted of some Latin America BHV-4 strains including MGArom, MGA696, etc. ${ }^{4,6,7,10}$

In Turkey, many serological investigations on BHV-4 have been performed, however, studies are limited and which genotypes have been circulated is unknown. ${ }^{11-17}$ In this study, therefore, it was aimed to investigate the potential presence of $\mathrm{BHV}-4$ in a dairy cattle herd with reproductive problems (repeat breeding, infertility, postpartum infection). It was additionally intended to elucidate current molecular evolutionary status by phylogenetic and recombination analysis.

\section{Materials and methods}

\section{Materials}

Uterine tract swabs were taken from 25 animals which have been consistently possessed reproductive problems (repeat breeding, infertility, postpartum infection) in a dairy herd. This small family dairy management had 67 cattle and located in Bozdogan, Aydin, province of the Southern Aegean region of Turkey. The ages of sampled animals ranged from two to five years that had reproductive problems in their sets in PCR that could amplify partial gB and TK history. Animals were only vaccinated with vaccines applied genes were modified based on reports of Wellenberg et by public veterinary services. Vaccines against BVDV, al. ${ }^{19}$ and Verna et al. ${ }^{6}$, respectively. PCR protocols were $\mathrm{BLV}$, and, $\mathrm{BHV}-1$ have not included in the mentioned presented as follows: initial denaturation $\left(95^{\circ} \mathrm{C} 5 \mathrm{~min}\right)$; vaccination program. All animals, except one, were healthy- amplification $\left(94^{\circ} \mathrm{C} 30 \mathrm{~s}\right.$, variable to $\mathrm{Tm}$ of primer looking in the clinical observation. The mentioned animal pairs $\left.30 \mathrm{~s}, 72^{\circ} \mathrm{C} 50 \mathrm{~s}\right)$ for 35 cycles; final extension $\left(72^{\circ}\right.$ had some clinical signs, which were hemorrhagic lesions C $7 \mathrm{~min}$ ). Purified amplicons were sequenced by Sanger around the mouth and muzzle area, anorexia, dehydration, method. Sequences were cleaned from noisy and weak diarrhea, pyrexia (Figure 1). Whole blood sample and nasal traces by using a software (Mega 10.0)20. To confirm the swab were also collected from this animal. This herd had information of negativity of BHV-1, BLV and BVDV, been investigated by PCRs in a private diagnostic we also performed PCR tests by each relevant primer laboratory in terms of various reproductive infection pairs that globally used..$^{21-23}$ agents including Bovine Viral Diarrhea Virus (BVDV),

Obtained sequence data batch were submitted to GenBank database by interface Banklt. Accession numbers have been assigned. 
(TR/BHV-4/2018/Bozdogan1-TK $\rightarrow$ MN173774; TR/ BHV-4/2018/Bozdogan2-TK $\rightarrow$ MN173775; TR/ BHV-4/2018/Bozdogan1-g(B) $\rightarrow$ MN173776; TR/ BHV-4/2018/Bozdogan2-g(B) $\rightarrow$ MN173777) and were presented in phylogenetic trees (Figure 2; 3). For phylogenetic analysis, relevant reference strain sequences were downloaded from GenBank. To construct maximum-likelihood phylogenetic tree, HasegawaKishino-Yano (HKY) and Tamura 3 (T92) models were determined as best model for $\mathrm{gB}$ and TK genes, respectively. 1000 bootstrap replicates were chosen for optimal calculation during tree construction.

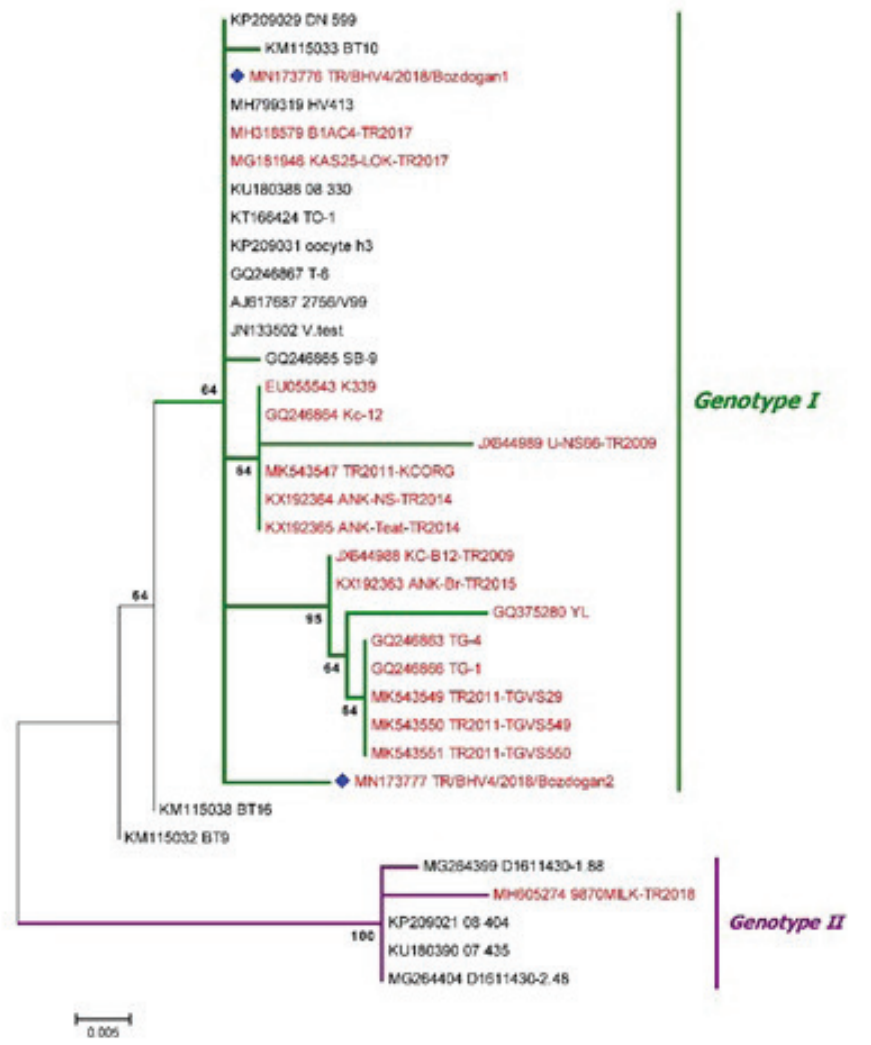

Figure 2: Maximum Likelihood tree based on glycoprotein B (gB) were constructed using Hasegawa-Kishino-Yano model with 1000 bootstrap replicates. All Turkish sequences were determined in red-colored and novel Turkish sequences have been also marked with “" . All Turkish sequences except one fell into Genotype I. TR/BHV-4/2018/Bozdogan2 distantly localized to TR/BHV-4/2018/Bozdogan1 in this genotype.

Recombination detection program, RDP4, was used to reveal potential recombination in $\mathrm{gB}$ and TK gene. Herein, also, the distance plot algorithm has been run with representative strains from genotypes and obtained sequences in this study (Figure 4).

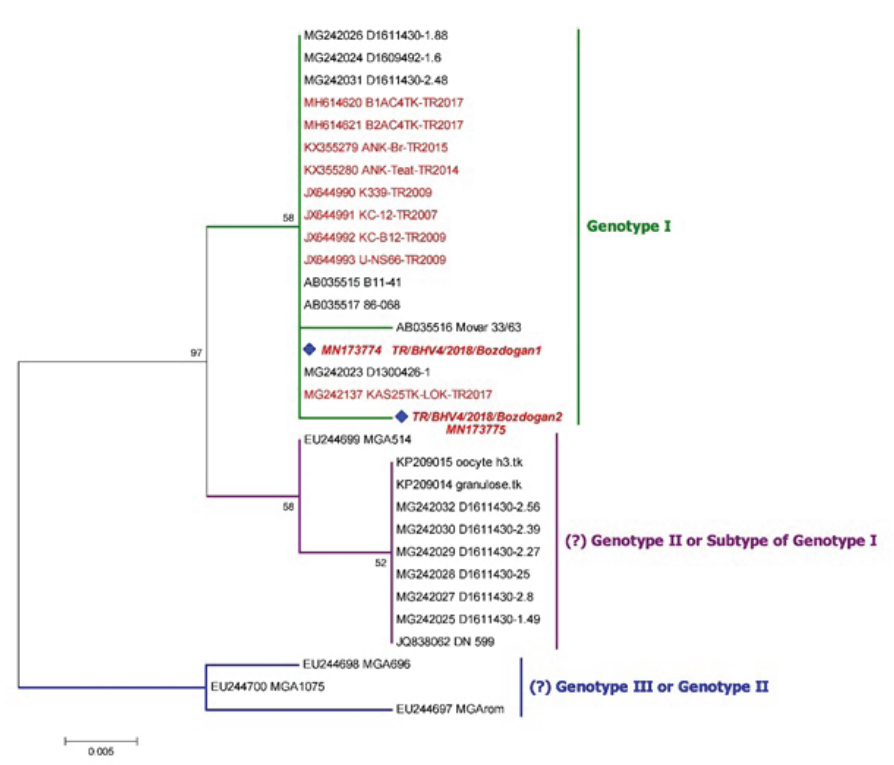

Figure 3: Maximum Likelihood tree based on thymidine kinase (TK) were constructed using Tamura 3 model with 1000 bootstrap replicates. All Turkish sequences were determined in red-colored and novel Turkish sequences have been also marked with “". Three main genotypes have occurred and all Turkish sequences monophyletically localized to each other into Genotype I.

\section{A}

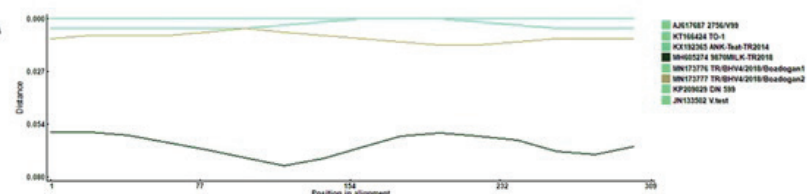

B

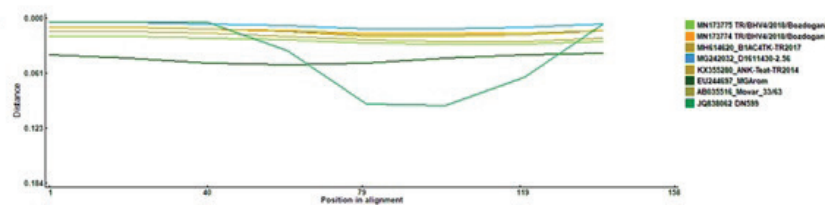

Figure 4: Distance plot analysis based on both glycoprotein B (gB) and thymidine kinase (TK) in RDP4. [A] Distance plot based on glycoprotein B (gB) [B] Distance plot based on thymidine kinase (TK)

\section{Results}

BHV-1, BVDV and BLV were found to be negative in all samples. Two out of twenty-five animals have been found BHV-4 positive, and both gene amplicons were available for phylogenetic and recombination assessment. Only vag-inal swabs were found positive, on the other hand other samples (nasal and blood samples) were negative. After se-quence analysis, obtained sequences were named as TR/BHV-4/2018/Bozdogan1 and TR/ BHV-4/2018/Bozdo-gan2. Both sequences (Bozdogan1 and Bozdogan2) were found 95\%-99.7\% identical to other BHV-4 sequences in GenBank database by using BLAST interface.

In phylogenetic assessment, both sequences monophyle- 
In $\mathrm{gB}$ ML unrooted tree, TR/BHV-4/2018/Bozdogan1 located closer to prior Turkish and prominent reference sequences, whereas TR/BHV-4/2018/ Bozdogan2 has drawn a separated branch in Gen-otype 1 (Figure 2). TK gene-based unrooted ML tree has generated similar tree topology, but this tree was separated into three major genotypes (Figure 3).

In RDP4, there was no recombinational evidence between new and reference sequences, available in GenBank, based on both gB and TK region. No unique changes have been found in toggle translation, however, distance plot based on nucleotide identities has indicated nucleotide differences (Figure 4). TR/BHV-4/2018/

Bozdogan1 had no shown any clinical symptoms, while TR/BHV-4/2018/Bozdogan2 had clinical symptoms including hemorrhagic lesions around the mouth and muzzle area, anorexia, dehydration, diarrhea, pyrexia.

\section{Discussion}

BHV-4 is one of the viral agents which has been substantially assumed to trigger reproductive problems in cattle all around the world. Despite this assumption, a wide variety of clinical symptoms including dermatitis, mastitis, vascu-litis, respiratory tract disorders, has also been declared in previous reports by some researchers. ${ }^{1-7,11-17}$ One of them, a case report by Bellino et. $\mathrm{al}^{4}$ claimed that BHV-4 might likely to be the reason for Dermatitis-Pyrexia-Hemorrhagic Syndrome (DPHS) in a cow with relevant symptoms. Intriguingly,

BHV-4/2018/Bozdogan2 similarly had TR/ many of mentioned symptoms in the cow with DPHS although blood and nasal swab samples were negative in PCR test. This leads to be thought that BHV-4 proportionally causes reproductive problems rather than other symptoms as it stated before. ${ }^{1,711,12,15-17}$ Pathoclinical linking remains to be elucidated by further detailed analysis. BHV-4 might also be a triggering factor providing to be occurred relevant symptoms by major subclinical diseases. Therefore, exhibiting of the accurate mechanism for BHV-4 can be provided by various pathoclinical studies.

Existence of a wide variety of clinical symptoms of BHV-4 have leads to be investigated potential genetic diversity by comparing BHV-4 sequences from this study and Gen-Bank database. Within this aim, phylogenetic and recom-bination analyses have been carried out. gB and TK gene regions were focused due to a wide sequences data is available in Genbank.
Alignment results indicated that there were no significant changes between BHV-4 sequences, however, it has been observed some nucleotide substitutions that might be effective on translational level. (Figure4). Notwithstanding, these point changes in BHV-4 nucleotide level have induced a putative genotyping in phyloge-netic tree in both this and previous studies ${ }^{4,6,10,25}$. Diversity and genotyping have been proportionally observed much more in TK based ML phylogenetic tree (Figure 3). gB has been mainly focused as recognizable region in BHV-4 lab-detection because of it is more conserved region, thus, it has less genotypes rather than TK based tree in phyloge-netic assessment of this study (Figure 2). gB and TK tree had two and three major genotypes, respectively. In both phylogenetic trees, novel Turkish BHV-4 sequences of this study have localized in Genotype I, but TR/BHV-4/2018/Bozdogan2 has drawn a separated branch in this genotype. Surprisingly, diversity of nucleotide and distance rate in plot analysis between two sequences were more significant in $\mathrm{gB}$ based tree. Additionally, DN-599, the reference strain originated from America, was localized under Genotype I in $\mathrm{gB}$ phylogenetic tree, whereas, in TK tree was involved into Genotype II. Howsoever, novel Turkish BHV-4 se-quences for this study have been involved into Genotype I as a sister taxon for European BHV-4 strains in both con-structed trees (Figure 2;3). If this output has been com-bined by prior obtained results from Turkey, it might be claimed that BHV-4 strains belonging to Genotype I have predominantly circulated in Turkey. ${ }^{11,12,26}$

Conducted phylogenetic trees and occurred genotypes in this study were in accordance with proposed genotyping model from the previous prominent reports. ${ }^{4,6,710}$ In con-trast, a recent study by Areda et al..$^{25}$ mentioned three and two genotypes for $\mathrm{gB}$ and $\mathrm{TK}$, respectively, in their phy-logenetic study. This indicates that deep sequencing and detailed phylogenetic analyses, including different gene regions, needs to be performed for the accurate knowledge on BHV-4 genotyping and recombination analysis.

To the author's knowledge, one study which was presented in 2013 reported genotypes of BHV-4 circulating among Turkish cattle for the first time ${ }^{26}$. All molecular data from the mentioned prior report is compatible with the results of this study. Both studies have revealed that almost all Turkish BHV-4 sequences belonged to Genotype I. ${ }^{11-17,26}$ Molecular data from previous studies were limited and not eligible rather than serological ones, thus, its comparison could not be comprehensively conducted in phylogenetic ${ }_{64}$ and recombination analyses. 


\section{Conclusion}

According to results, Turkish BHV-4 sequences were predominantly clustered in Genotype I, however, some nucleotide changes existed. Collected materials should be subjecteds to next-generation sequencing, through by, quite a knowledge on both nucleotide and amino acid data will provide the most accurate evaluation. BHV-4 might possess a highly importance as the potential agent for the non-defined reproductive diseases, however, its genetic structure and pathogenesis have not been already clear. This limits to develop novel strategies for struggle and prevention to BHV-4 related reproductive diseases. Therefore, knowledge of genetic structure and prevalence of BHV-4 should be consistently updated, and BHV-4 should be strictly taken into account for the diagnosis of reproductive diseases.

\section{Acknowledgment}

The author thanks to M.Ç., regional veterinarian, for all support in collection of samples. This study does not contain any studies involving experimental animals and present any ethical concerns. It has been registered by Local Ethical Committee for Experimental Animals (no.64583101/2019/008). All client approved and signed the informed consent form that allowing to use of collected samples as materials for this study. This study is self-funded.

\section{Declaration on Conflict of interest}

The author declares no conflict of interest.

\section{References}

1) Chastant-Maillard S. Impact of bovine herpesvirus 4 (BoHV-4) on reproduction. Transbound Emerg Dis. 2015;62(3):245-251. doi:10.1111/tbed.12155

2) Bartha A, Juhasz $M$, Liebermann $H$. Isolation of a bovine herpesvirus from calves with respiratory disease and keratoconjunctivitis. A preliminary report. Acta Vet Acad Sci Hung. 1966;16(3):357-358.

3) Davison AJ. Herpesvirus systematics. Vet Microbiol. 2010;143(1):52-69. doi:10.1016/j.vetmic.2010.02.014

4) Bellino C, Iussich S, Biasato I, et al. Potential pathogenetic role of bovine herpesvirus 4 in two dairy cows with dermatitis-pyrexia-hemorrhagic syndrome. J Clin Microbiol. 2015;53(8):2763-7. doi:10.1128/ JCM.00717-15

5) Egyed L. Bovine herpesvirus type 4: a special herpesvirus (review article). Acta Vet Hung. 2000;48(4):501doi:10.1556/004.48.2000.4.13

6) Verna AE, Manrique JM, Perez SE, et al. Genomic analysis of bovine herpesvirus type 4 (BoHV-4) from Argentina: high genetic variability and novel phylogenetic groups. Vet Microbiol. 2012;160(1-2):1-8. doi:10.1016/j.vetmic.2012.04.039
7) Gagnon CA, Traesel CK, Music N, et al. Whole genome sequencing of a canadian bovine gammaherpesvirus 4 strain and the possible link between the viral infection and respiratory and reproductive clinical manifestations in dairy cattle. Front Vet Sci. 2017;4:92. doi:10.3389/fvets.2017.00092

8) Zimmermann W, Broll H, Ehlers B, et al. Genome sequence of bovine herpesvirus 4 , a bovine rhadinovirus, and identification of an origin of DNA replication. J Virol. 2001;75(3):1186-94. doi:10.1128/

JVI.75.3.1186-1194.2001

9) Rasheed MA, Ansari AR, Ihsan A, et al. Prediction of conserved sites and domains in glycoproteins B, C and D of herpes viruses. Microb Pathog. 2018;116:91-99.

10) Morán $P$, Pérez $S$, Odeón $A$, et al. Comparative analysis of replicative properties of phylogenetically divergent, Argentinean BoHV-4 strains in cell lines from different origins. Comp Immunol Microbiol Infect Dis. 2019;63:97-103. doi:10.1016/j.cimid.2019.01.014

11) Bilge-Dagalp S, Demir AB, Gungor E, et al. The seroprevalence of Bovine Herpes Virus Type 4 (BHV-4) infection in dairy herds in Turkey and possible interaction with reproductive disorders. Rev Med Vet (Toulouse). 2007;158(4):201.

12) Bilge-Dagalp $S$, Gungor E, Demir AB, et al. The investigation of the presence of bovine herpesvirus type 4 (BoHV-4) in cows with metritis in a dairy herd. Ankara Univ Vet Fak Derg. 2010;57(2):87-91.

13) Gür S. Prevalence of bovine viral diarrhoea, bovine herpesvirus type 1 and 4 infections in repeat breeding cows in Western Turkey. Braz J Vet Res Anim Sci. 2011;48(3):228-233.

14) Aslan ME, Azkur AK, Gazyagci S. Epidemiology and genetic characterization of BVDV, BHV-1, BHV-4, BHV-5 and Brucella spp. infections in cattle in Turkey. J Vet Med Sci. 2015;14-0657.

15) Kale M, Ata A, Kocamüftüoglu M, et al. Bovine herpesvirus type 4 (BHV-4) infection in relation to fertility in repeat breeder dairy cows. Acta Vet (Beograd). 2011;61(1):13-19.

16) Yavru $S$, Avci $O$, Kale M, et al. Serologic and virologic investigation of BHV-1, BVDV and BHV-4 in cattle with metritis. Anim Vet Sci. 2014;2(5):142-145. 
17) Yildirim Y, Yilmaz V, Kalaycioglu AT, et al. An investigation of a possible involvement of BVDV, BHV-1 and BHV-4 infections in abortion of dairy cattle in Kars district of Turkey. Kafkas Univ Vet Fak Derg. 2011;17:879-883.

18) Chomczynski P, Sacchi N. The single-step method of RNA isolation by acid guanidinium thiocyanate-phenol-chloroform extraction: twenty-something years on. Nat Protoc. 2006;1(2):581.

19) Wellenberg GJ, Verstraten ERAM, Belak $S$, et al. Detection of bovine herpesvirus 4 glycoprotein $\mathrm{B}$ and thymidine kinase DNA by PCR assays in bovine milk. J Virol Meth. 2001;97(1-2):101-112.

20) Kumar S, Stecher G, Li M, et al. MEGA X: molecular evolutionary genetics analysis across computing platforms. Mol Bio Evol. 2018;35(6):1547-1549.

21) Vilcek S, Durkovic B, Kolesárová,M, et al. Genetic diversity of international bovine viral diarrhoea virus (BVDV) isolates: identification of a new BVDV-1 genetic group. Vet Res. 2004;35(5):609-615.

22) Fuchs M, Hübert P, Detterer J, et al. Detection of bovine herpesvirus type 1 in blood from naturally infected cattle by using a sensitive PCR that discriminates between wild-type virus and virus lacking glycoprotein E. J Clin Microbiol. 1999;37(8):2498-2507.

23) Santos MJD, Trono K, Lager I, et al. Development of a PCR to diagnose BLV genome in Frozen semen samples. Vet Microbiol. 2007;119:10-18.

24) Martin DP, Murrell B, Golden M, et al. RDP4: Detection and analysis of recombination patterns in virus genomes. Virus Evol. 2015;1(1).

25) Areda D, Chigerwe M, Crossley B. Bovine herpes virus type-4 infection among postpartum dairy cows in California: risk factors and phylogenetic analysis. Epidemiol Infect. 2018;146(7):904-912.

26) Bilge-Dağalp S, Babaoğlu AR, Doğan F, Timurkan MÖ, Oğuzoğlu TÇ, Özkul A, Alkan, F. Genomic Analysis of Bovine Herpesvirus Type 4 (BoHV-4) From Turkey. 4 th ESVV Veterinary Herpesvirus Symposium, Zürich, Switzerland, 2013, Poster Presentation. 